\title{
Instrumentação para espectroscopia de ressonância de ultrassom
}

\section{(Instrumentation for resonant ultrasound spectroscopy)}

\author{
R. F. de L. Lorenzi, J. E. Zorzi, C. A. Perottoni \\ Instituto de Materiais Cerâmicos, Universidade de Caxias do Sul, Bom Princípio, RS, Brasil 95765-000 \\ jezorzi@ucs.br
}

\begin{abstract}
Resumo
A técnica de espectroscopia de ressonância de ultrassom possibilita a determinação simultânea de todos os componentes do tensor de elasticidade do material que constitui a amostra. Neste trabalho é descrita a montagem de um dispositivo de espectroscopia de ressonância de ultrassom automatizado, que possibilita a análise de pequenas amostras na forma de paralelepípedos, cilindros ou esferas. A aplicação deste dispositivo é exemplificada com a determinação das constantes elásticas de amostras policristalinas de alumina e níquel. Os resultados obtidos diferem dos valores esperados em, no máximo, 2,6\% e 0,91\% para a alumina e níquel, respectivamente, e ilustram a aplicação deste dispositivo na determinação das constantes elásticas de pequenas amostras.
\end{abstract}

Palavras-chave: espectroscopia de ressonância de ultrassom, alumina, níquel.

\begin{abstract}
The resonant ultrasound spectroscopy technique (RUS) allows the simultaneous determination of all components of the tensor of elasticity for the material that constitutes the sample. In this work, an automated device for ultrasonic resonance spectroscopy was assembled which enables the analysis of small samples in the form of parallelepipeds, cylinders or spheres. The application of this device is exemplified by the determination of the elastic constants of polycrystalline samples of alumina and nickel. The results differ from the expected values by, at most, $2.6 \%$ and $0.91 \%$ for alumina and nickel, respectively, and demonstrate the usefulness of this device in determining the elastic stiffness of small samples.

Keywords: resonant ultrasound spectroscopy, alumina, nickel.
\end{abstract}

\section{INTRODUÇÃO}

A resposta de um sólido sujeito a um campo de tensões no regime linear elástico, depende de propriedades fundamentais do material que o compõe, particularmente do potencial interatômico. As propriedades elásticas de um material são também relacionadas às propriedades termodinâmicas, incluindo a capacidade calorífica a pressão constante, o coeficiente de expansão térmica e a temperatura de Debye $[1,2]$.

De modo geral, a deformação $(\varepsilon)$ exibida por um material anisotrópico (por exemplo, um monocristal) sujeito a um campo de tensões $(\sigma)$ segue a lei de Hooke generalizada [3-5]:

$$
\sigma_{\mathrm{ij}}=\mathrm{C}_{\mathrm{ijkl}} \varepsilon_{\mathrm{kl}}
$$

na qual $c$ é o tensor de elasticidade e os índices $i, j, k, l$ podem ser 1, 2 ou 3 (na expressão acima aplica-se a conversão da soma sobre índices repetidos). As componentes com $i=j$ são denominadas tensões normais ou tensões de tração (com a compressão considerada uma tensão de tração negativa). No caso das tensões normais, as forças agem na direção perpendicular (normal) à área sobre a qual são aplicadas. As componentes com $i \neq j$ são chamadas tensões de cisalhamento e, nestas, as forças atuam em uma direção paralela à área sobre a qual são aplicadas [3,4]. Tanto a tensão aplicada quanto a deformação resultante são grandezas tensoriais de segunda ordem e, conseqüentemente, o tensor de elasticidade é um tensor de quarta ordem. Portanto, em princípio, são necessários 81 componentes para descrever completamente o comportamento linear elástico de um material [3]. No entanto, relações de simetria $\left(\sigma_{i j}=\sigma_{j i}\right.$ para $\left.i \neq j\right)$ permitem reduzir o número de constantes elásticas para 36 , que podem ser agrupadas em uma matriz 6 × 6 [3]. De fato, usando a notação de Voigt [3], a expressão (A) pode ser reescrita como

$$
\sigma_{\mathrm{i}}=\mathrm{C}_{\mathrm{ij}} \varepsilon_{\mathrm{j}}
$$

na qual os índices $i j$ e $k l$ da expressão (A) são alterados de acordo com a convenção [3]:

$$
\begin{gathered}
11 \rightarrow 122 \rightarrow 233 \rightarrow 3 \\
23(\text { ou } 32) \rightarrow 413(\text { ou } 31) \rightarrow 512(\text { ou } 21) \rightarrow 6
\end{gathered}
$$

O número de constantes elásticas $c_{i j}$ independentes pode ser ainda mais reduzido levando em consideração a classe de simetria. Por exemplo, o número de constantes elásticas independentes é igual a 21 no caso de cristais de simetria triclínica, 3 para cristais de simetria cúbica e 2 para materiais 
isotrópicos [3,6]. Neste último caso, um material homogêneo e isotrópico tem seu comportamento no regime linear elástico definido por dois (de quatro) módulos elásticos de engenharia: o módulo de Young ( $E$, que expressa a resistência oferecida pelo material à deformação elástica paralela à direção de aplicação da tensão), o módulo volumétrico ( $B$, que é o inverso da compressibilidade isotérmica), o módulo de cisalhamento ( $\mu$, que é uma medida da resistência que o material impõe a tensões cisalhantes) e a razão de Poisson ( $v$, que é o negativo da razão entre a deformação transversal e a deformação longitudinal paralela à tração). Em um material homogêneo e isotrópico estes quatro módulos elásticos de engenharia se relacionam através de [3]:

$$
\begin{aligned}
& E=3 B(1-2 v)=2 \mu(1+v)=\frac{9 \mu B}{3 B+\mu} \\
& \mu=\frac{3 E B}{9 B-E}=\frac{E}{2(1+v)}=\frac{3 B(1+2 v)}{2(1+v)} \\
& v=\frac{3 B-E}{6 B}=\frac{E-2 \mu}{2 \mu}=\frac{3 B-2 \mu}{2(3 B+\mu)} \\
& B=\frac{\mu E}{3(3 \mu-E)}=\frac{2 \mu(1+v)}{3(1-2 v)}=\frac{E}{3(1-2 v)}
\end{aligned}
$$

Por sua vez, os módulos elásticos de engenharia de uma amostra homogênea e isotrópica são calculados a partir das constantes elásticas independentes $\mathrm{c}_{11}$ e $\mathrm{c}_{12}$ através de

$$
\begin{aligned}
& \mathrm{c}_{11}=\mathrm{B}+\frac{4}{3} \mu_{0} \\
& \mathrm{c}_{12}=\mathrm{B}+\frac{2}{3} \mu_{0}
\end{aligned}
$$

A determinação simultânea de todas as constantes elásticas independentes em um único experimento é uma das características mais interessantes da técnica de espectroscopia de ressonância de ultrassom (RUS, da sigla em inglês para resonant ultrasound spectroscopy). De fato, as freqüências de ressonância de um sólido podem ser determinadas a partir das suas dimensões, massa e tensor de elasticidade. No entanto, na técnica de RUS temos o problema inverso: as freqüências de ressonância são medidas experimentalmente, junto com as dimensões físicas e a massa da amostra e, a partir destas informações, o tensor de elasticidade é obtido por meio de um processo iterativo $[7,8]$.

Estudos recentes apontaram a superioridade da técnica de RUS (comparada, por exemplo, com o tradicional ensaio de flexão de quatro pontos) na determinação das propriedades elásticas de materiais policristalinos [9]. A simplicidade da técnica, no entanto, tem como contraponto a complexidade do processo do cálculo do tensor de elasticidade da amostra a partir do seu espectro RUS. A solução para este problema, no entanto, tem sido implementada em programas de computador que permitem a análise de monocristais de materiais com classe de simetria cúbica a ortorrômbica, além de amostras policristalinas isotrópicas [10].

De modo geral, um dispositivo para realizar medidas de espectroscopia de ressonância de ultrassom, utiliza um transdutor piezoelétrico para excitar ressonâncias mecânicas em uma amostra e outro para detectá-las (Fig. 1). A instrumentação consiste basicamente de um gerador de sinal (cuja freqüência possa ser variada de maneira controlada) e um amplificador/detector lock-in. A amostra geralmente possui a forma de paralelepípedo ou cilindro e tem dimensões que variam de $100 \mu \mathrm{m}$ até alguns poucos centímetros.

Neste trabalho será descrita a montagem de um dispositivo para medição das constantes elásticas de materiais por espectroscopia de ressonância de ultrassom. Como ilustração da aplicação desta técnica, o dispositivo experimental será utilizado na determinação das constantes elásticas independentes de amostras de alumina e de níquel.

\section{MATERIAIS E MÉTODOS}

O dispositivo para RUS utiliza dois transdutores piezoelétricos: um para excitar ressonâncias mecânicas na amostra e outro para detectá-las (Fig. 1). A instrumentação eletrônica consiste essencialmente de um gerador de sinal e um amplificador/detector lock-in, ambos controlados por um computador, que é responsável também pela aquisição de dados.

Montagem do sistema de espectroscopia de ressonância de ultrassom

Para a montagem do equipamento de espectroscopia de ressonância de ultrassom foram utilizados um computador

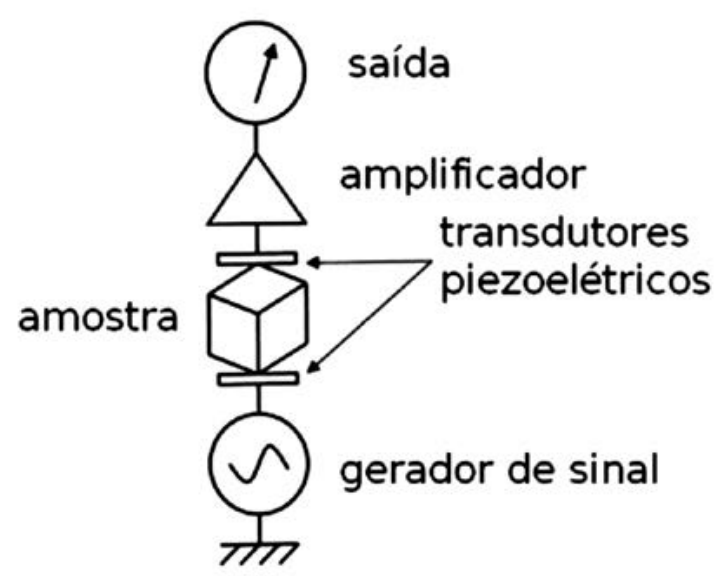

Figura 1: Representação esquemática de um dispositivo para espectroscopia de RUS. Adaptado de [11].

[Figure 1: Schematic representation of a device for RUS spectroscopy. Adapted from [11].] 
com o software LabView (programa para automatizar o processo de medição), duas placas GPIB-USB-HS (para comunicação entre o computador e os equipamentos), um gerador de sinal DS345 (Stanford Research), um amplificador lock-in SR844 (Stanford Research) e um dispositivo para posicionar a amostra entre os transdutores piezoelétricos. $\mathrm{O}$ amplificador lock-in SR844 opera de $25 \mathrm{kHz}$ até $200 \mathrm{MHz}$ e possui interfaces GPIB e RS-232, de modo que o processo de medida pode ser automatizado [12]. O gerador de sinal DS345 atua na faixa de freqüência que vai de $1 \mu \mathrm{Hz}$ a 30,2 $\mathrm{MHz}$, com resolução de $1 \mu \mathrm{Hz}$ e amplitude de 0,1 a $10 \mathrm{Vpp}$. $O$ gerador de sinal conta também com interface GPIB e RS232 [13].

\section{Transdutores piezoelétricos}

Foram utilizados transdutores piezoelétricos convencionais de PZT (titanato zirconato de chumbo), no formato de discos com $8 \mathrm{~mm}$ de diâmetro e $2 \mathrm{~mm}$ de espessura. Uma camada de cola epóxi foi utilizada para fixar o transdutor piezoelétrico no interior de um conector BNC. Cola prata foi utilizada para fazer os contatos elétricos. $\mathrm{Na}$ Fig. 2 podemos ver um esquema da montagem do transdutor elétrico no interior de um conector bnc e duas fotografias do transdutor piezoelétrico após montagem.

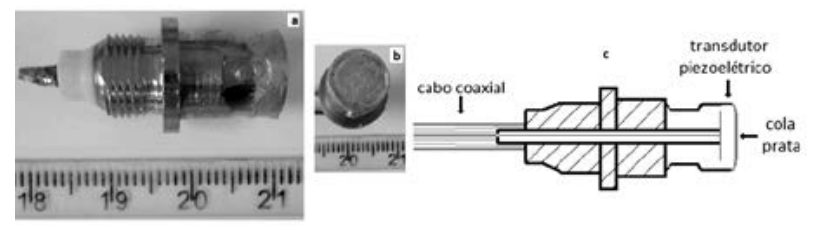

Figura 2: Em a e b duas vistas do sistema montado para fixar o transdutor piezoelétrico e em c, esquema da montagem do transdutor piezoelétrico sobre o conector BNC. A parte interna do cabo coaxial é colada com cola prata na parte inferior do transdutor piezoelétrico, enquanto a malha do cabo coaxial é colocada em contato elétrico com a carcaça do conector $\mathrm{BCN}$ e com a parte superior do transdutor.

[Figure 2: In $\boldsymbol{a}$ and $\boldsymbol{b}$ two different views of the system mounted to fix the piezoelectric transducer and c, scheme showing how the piezoelectric transducer is fixed on the BNC connector. The inner part of the coaxial cable is glued with silver paste at the bottom of the piezoelectric transducer, while the mesh of the coaxial cable is connected using silver glue to the ground BNC connector and to the top of the transducer.]

\section{Posicionamento da amostra}

A amostra deve ser suavemente apoiada entre os dois transdutores piezoelétricos (através dos vértices opostos da diagonal principal, no caso de amostras na forma de paralelepípedos), como ilustrado na Fig. 1. Para tanto, um posicionador xy foi adaptado à platina de um microscópio óptico, cujo ajuste de foco (ao longo do eixo z) permitiu um ajuste fino da força exercida pelos transdutores piezoelétricos sobre a amostra.

$\mathrm{Na}$ Fig. 3 podemos visualizar a montagem final do equipamento de espectroscopia de ressonância de ultrassom.

Quando a amostra entra em ressonância com o primeiro transdutor piezoelétrico, o sinal no segundo transdutor (receptor) apresenta um máximo. Para amostras com dimensões típicas, uma varredura de freqüência de 0,4 a 3 MHz é suficiente para detectar as freqüências de ressonância necessárias para calcular todo o tensor de elasticidade do material que compõe a amostra [10].

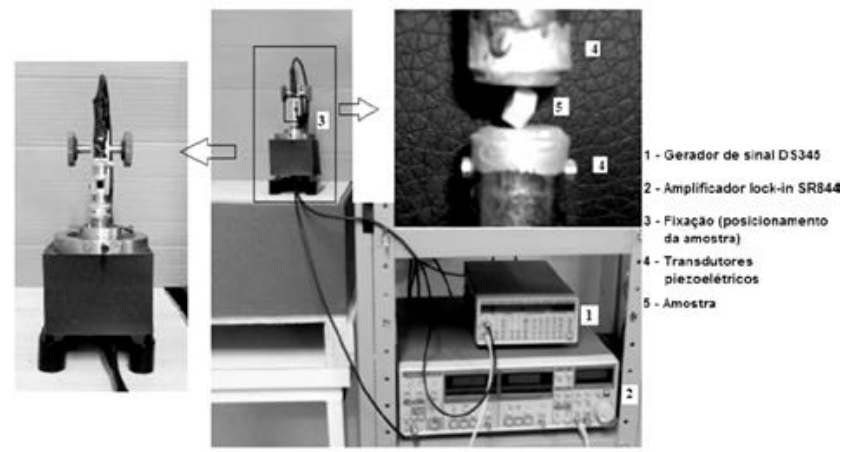

Figura 3: Dispositivo para espectroscopia de ressonância de ultrassom montado no IMC/UCS.

[Figure 3: Resonance ultrasound spectroscopy device assembled at IMC/UCS.]

\section{Amostra de alumina}

A amostra utilizada neste trabalho para testar o sistema de RUS foi preparada com alumina $\left(\mathrm{Al}_{2} \mathrm{O}_{3} \mathrm{~A}-1000 \mathrm{SG}\right)$ fornecida pela Almatis e foi confeccionada pela técnica de colagem de barbotina [14]. Após a conformação, a amostra foi pré-sinterizada a $1000^{\circ} \mathrm{C}$. Nesta etapa ocorre a formação de pescoço entre as partículas, que garante uma pequena resistência mecânica e possibilita o manuseio e acabamento da amostra. Após, a amostra foi sinterizada a $1600{ }^{\circ} \mathrm{C} / 2 \mathrm{~h}$ sobre leito de alumina A1 (Alcoa). Para realizar as medidas de espectroscopia de ressonância por ultrassom, a amostra de alumina foi cortada na forma de um paralelepípedo em uma cortadeira de precisão IsoMet 1000 (Buehler, Estados Unidos). Para o polimento, foi confeccionado um dispositivo para prender a amostra e garantir o paralelismo entre as faces opostas. Placas retangulares de vidro foram fixadas a uma base rígida com cola ativada termicamente (Crystalbond $囚)$ [6]. Após o corte e o polimento, a amostra de alumina apresentou o formato de um paralelepípedo com dimensões de $0,2456 \times 0,2412 \times 0,2079 \mathrm{~cm}$.

\section{Amostra de níquel}

O sistema de espectroscopia de ressonância de ultrassom foi testado também com uma amostra cilíndrica de níquel policristalino de alta pureza ((Kurt J. Lesker Co., Estados Unidos, 99,995\%). A amostra cilíndrica foi utilizada como recebida (diâmetro de $0,3167 \mathrm{~cm}$ e altura de $0,2500 \mathrm{~cm}$ ).

\section{Programas de controle, aquisição e análise de dados}

O programa utilizado para automatizar as medidas de 
RUS foi desenvolvido em LabView por Jon B. Betts (Los Alamos National Laboratory) [15]. Foram necessárias algumas alterações no programa para adequá-lo ao sistema descrito neste trabalho, já que a versão original foi escrita considerando um $h u b$ GPIB e, dessa forma, teria apenas um endereço para as placas GPIB no programa em LabView, diferenciando apenas os endereços do gerador de sinal e do amplificador lock-in. Já no sistema utilizado neste trabalho existem duas placas GPIB distintas e, portanto, são necessários dois endereços diferentes, um para cada placa GPIB e mais os endereços para cada equipamento [6]. O programa de controle e aquisição de dados gera um arquivo de saída com a freqüência e a amplitude do sinal medido no amplificador lock-in. Para determinar com precisão as freqüências de ressonância da amostra foi utilizado o programa XRDA [16], como ilustra a Fig. 4.

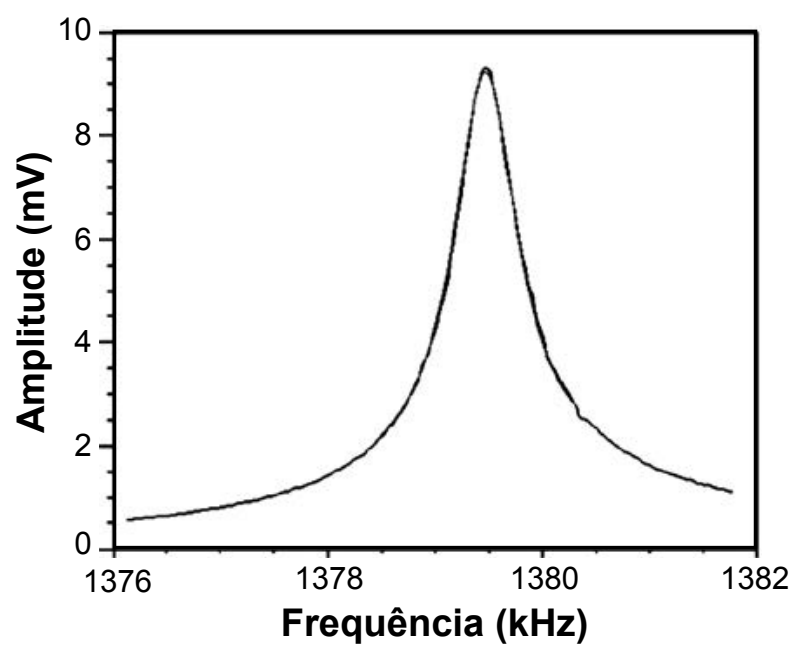

Figura 4: Ajuste de um perfil Lorentziano aos dados experimentais para a determinação precisa da freqüência de ressonância da amostra. No exemplo, a ressonância occore em 1379,48(4) kHz.

[Figure 4: Fitting of a Lorentzian profile to experimental data for the accurate determination of the sample's resonance frequency. In the example, the resonance occurs at 1379.48(4) $\mathrm{kHz}$.]

O uso da técnica de RUS implica num alto custo computacional para a solução do problema inverso, isto é, a obtenção do tensor de elasticidade a partir das freqüências de ressonância, dimensões físicas e massa da amostra. De fato, uma análise de RUS necessitava de aproximadamente $2 \mathrm{~h}$ por iteração no primeiro computador pessoal da IBM, 10 $\mathrm{s}$ no primeiro Cray e $0,8 \mathrm{~s}$ em um notebook com processador de $2 \mathrm{GHz}$ [15].

\section{Cálculo das freqüências de vibração}

Como dito anteriormente, a amostra a ser analisada é suavemente apoiada entre os dois transdutores piezoelétricos. Desta forma, os modos de vibração correspondem, aproximadamente, aos modos de vibração de um corpo livre. Uma solução analítica completa para o cálculo das vibrações livres de sólidos não existe, de modo que devemos recorrer a métodos aproximados. Destes, os métodos computacionais mais freqüentemente utilizados têm sido o método de elementos finitos e a técnica de minimização da energia. Esta última foi utilizada no programa de análise de dados empregado neste trabalho $[8,15]$.

Da mecânica clássica, a forma geral da Lagrangeana é

$$
L=\int_{V}(K E-P E) d V
$$

na qual $K E$ é a energia cinética e $P E$ é a energia potencial do sistema. Para um sólido elástico com volume $V$ e superfície livre $S$, com tensor de elasticidade $\mathrm{c}_{i j k l}$ e densidade $\rho$, a energia cinética é dada por

$$
\mathrm{KE}=\frac{1}{2} \sum_{\mathrm{i}} \rho \omega^{2} u_{i}^{2}
$$

na qual $\omega$ corresponde à freqüência de vibração e $u_{i}$ é o i-ésimo componente do vetor deslocamento. A energia potencial é, por sua vez, escrita como

$$
P E=\frac{1}{2} \sum_{i, j, k, 1} c_{i j k l} \frac{\partial u_{i}}{\partial_{x_{j}}} \frac{\partial u_{k}}{\partial_{x_{1}}}
$$

Usando o método de Rayleigh-Ritz, podemos expandir o vetor deslocamento em um conjunto completo de funções $\left\{\phi_{\lambda}\right\}$ :

$$
\mathrm{u}_{\mathrm{i}}=\sum_{\lambda} \mathrm{a}_{\mathrm{i} \lambda} \phi_{\lambda}
$$

A escolha de $\phi_{\lambda}$ é arbitrária. Migliori e Sarrao tomaram como função base $\phi_{\lambda}=x^{l} y^{m} z^{n}$, onde $\lambda=(l, m, n)$ é o rótulo da função, um conjunto de três inteiros não-negativos. Após substituição nas Equações J, K e I.

$\mathrm{L}=\int_{\mathrm{V}} \frac{1}{2}\left[\sum_{\mathrm{i}, \mathrm{i}, \lambda, \lambda} \delta_{\mathrm{ii}} \rho \omega^{2} a_{\mathrm{i} \lambda} a_{\mathrm{i} \lambda}, \phi_{\lambda} \phi_{\lambda,}, \sum_{\mathrm{i}, \mathrm{j}, \mathrm{k}, 1 \lambda, \lambda} \mathrm{c}_{\mathrm{ijk}} a_{\mathrm{i} \lambda} a_{\mathrm{i}, \lambda}, \frac{\partial \phi_{\lambda}}{\partial \mathrm{x}_{\mathrm{j}}} \frac{\partial \phi_{\lambda^{\prime}}}{\partial \mathrm{x}_{1}}\right] \mathrm{dV}$

na qual os somatórios de $\lambda$ e $\lambda$ ' são de 1 até $N$ e $\delta_{i i i^{\prime}}=1$ para $i$ $=i$ ' e 0 para $i \neq i$ '. A Equação $\mathrm{M}$ pode ser escrita em forma matricial como

$$
\mathrm{L}=\frac{1}{2} \omega^{2} \vec{a}^{\mathrm{T}} \overleftrightarrow{\mathrm{E}} \vec{a}-\frac{1}{2} \vec{a}^{\mathrm{T}} \stackrel{\leftrightarrow}{\Gamma} \vec{a}
$$

na qual $\vec{a}$ é o vetor de elementos $a_{i}$ cujo vetor transposto é $\vec{a}^{\mathrm{T}} \mathrm{e}$ $\overleftrightarrow{\mathrm{E}}$ e $\overleftrightarrow{\Gamma}$ são matrizes de ordem $R=3(N+1)(N+2)(N+3) / 6$, onde $l+m+n<N$. Para $N \rightarrow \infty$, a equação $\mathrm{M}$ fornece a solução exata para o problema. Na prática, $N$ deve ser restringido de forma que harmonize a precisão computacional e o 
tempo/memória de computação necessário. O truncamento da série resultará em autovalores que são maiores que os valores reais. No entanto, os erros resultantes diminuem monotonicamente com o aumento de $N$. Uma escolha razoável é $N=10$, dado os erros típicos de preparação de amostra.

A Lagrangeana é estacionária se os deslocamentos $u_{i}$ são soluções do problema da vibração livre. Estas soluções podem ser obtidas igualando a zero as derivadas da equação $\mathrm{M}$ com respeito a cada uma das $R$ amplitudes $a_{i \lambda}$. Este procedimento leva a uma equação de autovalores,

$$
\omega^{2} \overleftrightarrow{\mathrm{E}} \vec{a}=\overleftrightarrow{\Gamma} \vec{a}
$$

A matriz $\overleftrightarrow{\mathrm{E}}$ é simétrica e definida positiva e $\overleftrightarrow{\Gamma}$ é simétrica. Técnicas numéricas podem ser utilizadas para resolver a Equação (O). A solução para essa equação consiste nas freqüências de vibração livre do sólido e nos modos de vibração (autovetores) correspondentes.

O programa para análise dos dados de RUS utilizado neste trabalho foi desenvolvido por Albert Migliori (National High Magnetic Field Laboratory, Florida State University - Los Alamos National Laboratory - University of Florida), e encontra-se disponível no site da MagnetLab [17]. A versão atual permite determinar o tensor de elasticidade para materiais homogêneos e isotrópicos, além de monocristais com classe de simetria cúbica, hexagonal, tetragonal ou ortorrômbica. O programa desenvolvido por Migliori utiliza a equação $\mathrm{O}$ e, por meio do algoritmo de minimização de Levenberg-Marquard, encontra os valores das constantes elásticas independentes que fornece um conjunto de freqüências que mais se aproxima das freqüências de ressonância da amostra. Ao final, o programa lista as freqüências encontradas como solução da equação $\mathrm{O}$, juntamente com as freqüências de ressonância da amostra, determinadas experimentalmente. A comparação entre estes dois conjuntos de freqüências permite determinar modos de vibração que não tenham sido detectados experimentalmente e/ou freqüências de ressonância espúrias, não diretamente associadas à amostra. A estas freqüências associa-se um peso estatístico nulo, e o processo de ajuste por mínimos quadrados é reiniciado. Este processo é repetido até que a raiz quadrada da diferença média quadrática entre as freqüências calculadas e experimentais torne-se inferior a um valor pré-estabelecido (tipicamente 0,8\%) [15]. A presença de freqüências espúrias entre os resultados experimentais pode ser verificada reposicionando a amostra entre os transdutores piezoelétricos e repetindo as medições. A estimativa da incerteza típica nas medidas de RUS foi feita por simulação Monte Carlo. Para tanto, foram realizadas cinco medidas com a amostra de alumina. Para cada freqüência de ressonância, portanto, foram encontrados cinco valores distintos e, a partir deles, foram calculados a média e o desvio padrão. O passo seguinte foi gerar aleatoriamente (utilizando um gerador de números aleatórios com distribuição normal) vários conjuntos de freqüências de ressonância com média e desvio padrão iguais aos obtidos experimentalmente. Cada conjunto de freqüências foi utilizado para determinar $c_{11}$ e $c_{12}$, usando o programa para a análise de dados de RUS. O desvio padrão do conjunto de valores de $\mathrm{c}_{11}$ e $\mathrm{c}_{12}$ assim obtidos foi utilizado como estimativa da incerteza dos resultados.

\section{RESULTADOS E DISCUSSÃO}

O espectro de ressonância de ultrassom obtido para a amostra de alumina encontra-se representado na Fig. 5 (um exemplo de arquivo de entrada e de saída do programa utilizado para o cálculo das constantes elásticas da amostra de alumina usada neste trabalho encontra-se no Apêndice ao final deste artigo). Os resultados obtidos a partir da análise das freqüências de ressonância foram $c_{11}=452(8)$ $\mathrm{GPa}$ e $\mathrm{c}_{12}=141(8) \mathrm{GPa}$.

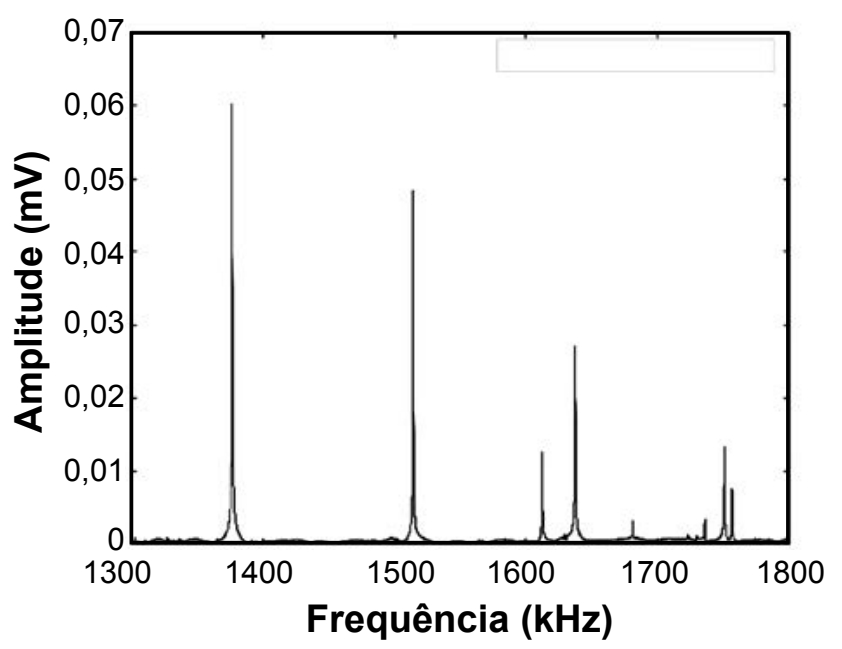

Figura 5: Espectro de ressonâncias para a amostra de alumina policristalina.

[Figure 5: Resonance spectrum for the polycrystalline alumina sample.]

Para uma melhor comparação com os resultados da literatura é necessário levar em conta o efeito da porosidade sobre as constantes elásticas da alumina. Alguns valores de

Tabela I - Dependência com a porosidade das constantes elásticas $\mathrm{c}_{11}$ e $\mathrm{c}_{12}$ de amostras policristalinas de alumina [18]. [Table I - Porosity dependent stiffness constants $c_{11}$ and $c_{12}$ for alumina polycrystalline samples [18].]

\begin{tabular}{|c|c|c|c|}
\hline$\rho\left(\mathrm{g} / \mathrm{cm}^{3}\right)$ & Porosidade $^{1}$ & $\mathrm{c}_{11}(\mathrm{GPa})$ & $\mathrm{c}_{12}(\mathrm{GPa})$ \\
\hline 3,94 & 0,011 & 478,6 & 162,9 \\
\hline 3,90 & 0,020 & 447,7 & 138,3 \\
\hline 3,82 & 0,041 & 426,8 & 139,2 \\
\hline 3,71 & 0,068 & 378,6 & 114,4 \\
\hline
\end{tabular}


$\mathrm{c}_{11}$ e $\mathrm{c}_{12}$ em função da porosidade (na faixa de valores de interesse para este trabalho), para alumina policristalina, são apresentados na Tabela I.

$\mathrm{O}$ efeito da porosidade é normalmente expresso em termos da relação entre a fração volumétrica de poros $P$ e os módulos elásticos. Existem várias propostas na literatura para a relação entre a porosidade e os módulos elásticos [4]. Em particular, foram propostas as seguintes expressões para o módulo volumétrico e o módulo de cisalhamento em termos da porosidade, sendo $B_{0}$ e $\mu_{0}$ os valores para porosidade nula $\left(B_{0}=256(6) \mathrm{GPa}\right.$ e $\mu_{0}=158(6) \mathrm{GPa}$ para a alumina $[4,18])$,

$$
\begin{aligned}
& \frac{1}{B}=\frac{1}{B_{0}(1-P)}+\frac{3 P}{4 \mu_{0}} 0\left(p^{3}\right) \\
& \mu=\mu_{0}\left[1 \frac{5\left(3 B_{0}+4 \mu_{0}\right)}{9 B_{0}+8 \mu_{0}} P\right]+0\left(\mathrm{p}^{2}\right)
\end{aligned}
$$

As Figs. 6 e 7 ilustram a relação entre as constantes elásticas $\mathrm{c}_{11}$ e $\mathrm{c}_{12}$ da alumina policristalina e a porosidade, obtida através das Equações G, H, P e Q, juntamente com os dados experimentais reportados [18].

$\mathrm{Na}$ Tabela II temos a comparação entre os valores medidos e os valores esperados para as constantes elásticas $c_{11}$ e $c_{12}$ da alumina (considerando $2 \%$ de fração volumétrica de poros, tal como estimada para a amostra utilizada neste trabalho). Nota-se uma boa concordância entre os resultados, apesar da dispersão observada nos valores reportados para a dependência de $c_{11}$ e $c_{12}$ com a porosidade da alumina.

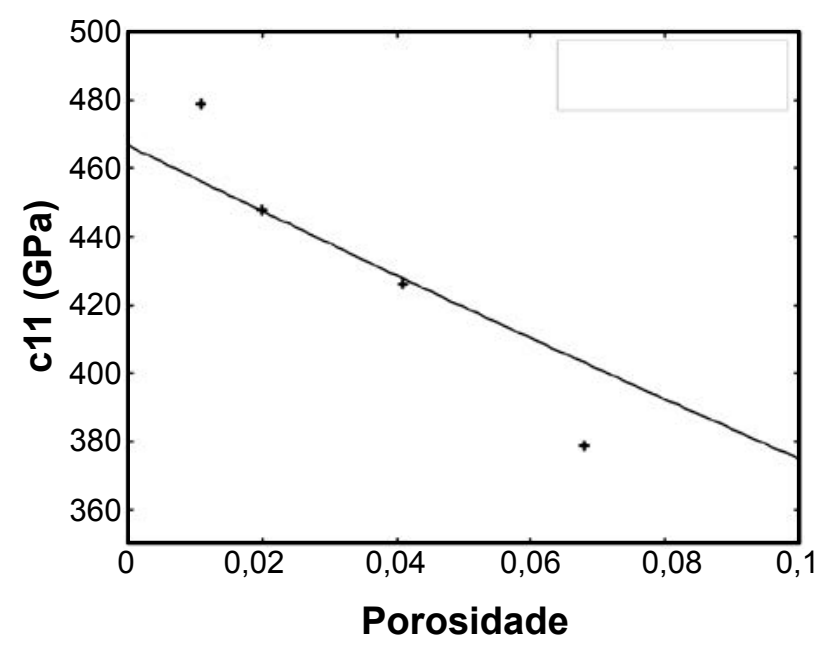

Figura 6: Constante elástica $\mathrm{c}_{11}$ da alumina policristalina em função da porosidade. A linha contínua representa o resultado obtido das expressões $(\mathrm{G}),(\mathrm{H}),(\mathrm{P})$ e $(\mathrm{Q})$. Os símbolos $(+)$ representam os dados reportados [18].

[Figure 6: $c_{11}$ elastic constant as a function of porosity for polycrystalline alumina. The continuous line represents the result obtained from expressions $(G),(H),(P)$ and $(Q)$. Symbols (+) represent reported data [18].]

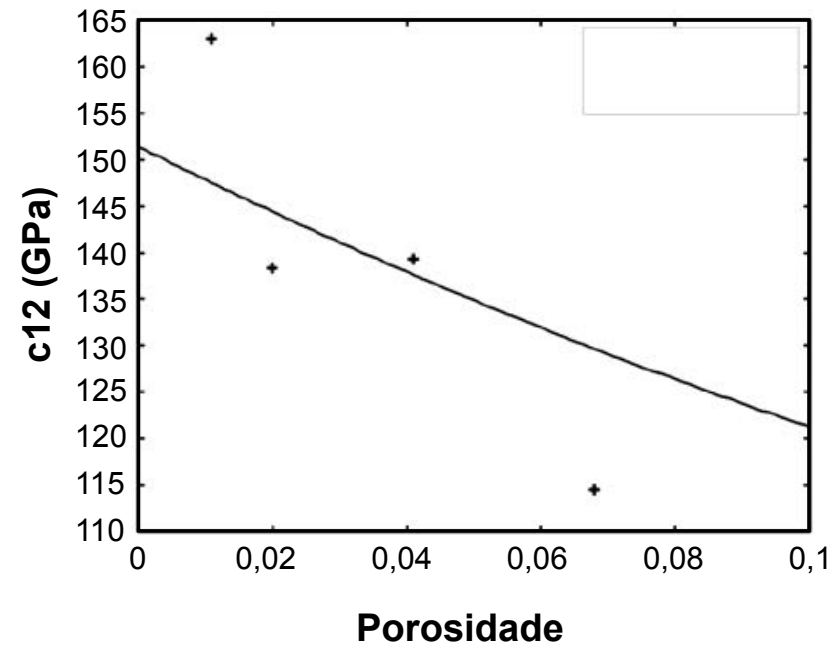

Figura 7: Constante elástica $c_{12}$ da alumina policristalina em função da porosidade. A linha contínua representa o resultado obtido das expressões $(\mathrm{G}),(\mathrm{H}),(\mathrm{P})$ e $(\mathrm{Q})$. Os símbolos $(+)$ representam os dados reportados [18].

[Figure 7: $c_{12}$ elastic constant as a function of porosity for polycrystalline alumina. The continuous line represents the result obtained from expressions $(G),(H),(P)$ and $(Q)$. Symbols (+) represent reported data [18].]

Tabela II - Comparação entre os resultados esperados e os valores determinados experimentalmente para as constantes elásticas de uma amostra de alumina com $2 \%$ de porosidade. [Table II - Comparison between expected and experimental values for the elastic constants of a $2 \%$ porosity alumina sample.]

\begin{tabular}{cccc}
\hline Constante elástica & $\mathrm{c}_{11}(\mathrm{GPa})$ & $\mathrm{c}_{12}(\mathrm{GPa})$ & $\mathrm{c}_{44}(\mathrm{GPa})^{1}$ \\
\hline Esperado $^{2}$ & $447(6)$ & $144(6)$ & $151(6)$ \\
Medido & $452(8)$ & $141(8)$ & $155(6)$ \\
Diferença (\%) & 1,1 & 2,1 & 2,6 \\
\hline
\end{tabular}

${ }^{1}$ Isotrópico, $\mathrm{c}_{44}=\frac{\mathrm{c}_{11-\mathrm{c}_{12}}}{2}$

${ }^{2}$ Valores esperados, com intervalo de confiança de lo como estimativa da incerteza de $c_{11}$ e $c_{12}$.

\section{Teste com níquel}

O procedimento experimental adotado para a amostra de níquel foi o mesmo empregado com a amostra de alumina. Na Fig. 8 e abaixo podemos visualizar o espectro de RUS resultante obtido com uma amostra cilíndrica de níquel.

Tabela III - Resultados da análise de RUS para a amostra policristalina de níquel.

[Table III - RUS analysis results for the sample of polycrystalline nickel.]

\begin{tabular}{cccc}
\hline Constante elástica & $\mathrm{c}_{11}(\mathrm{GPa})$ & $\mathrm{c}_{12}(\mathrm{GPa})$ & $\mathrm{c}_{44}(\mathrm{GPa})$ \\
Esperado & 312,1 & 125,7 & 93,2 \\
Medido & $312,6(8)$ & $126,8(8)$ & $92,9(8)$ \\
Diferença (\%) & 0,15 & 0,91 & 0,32 \\
\hline
\end{tabular}




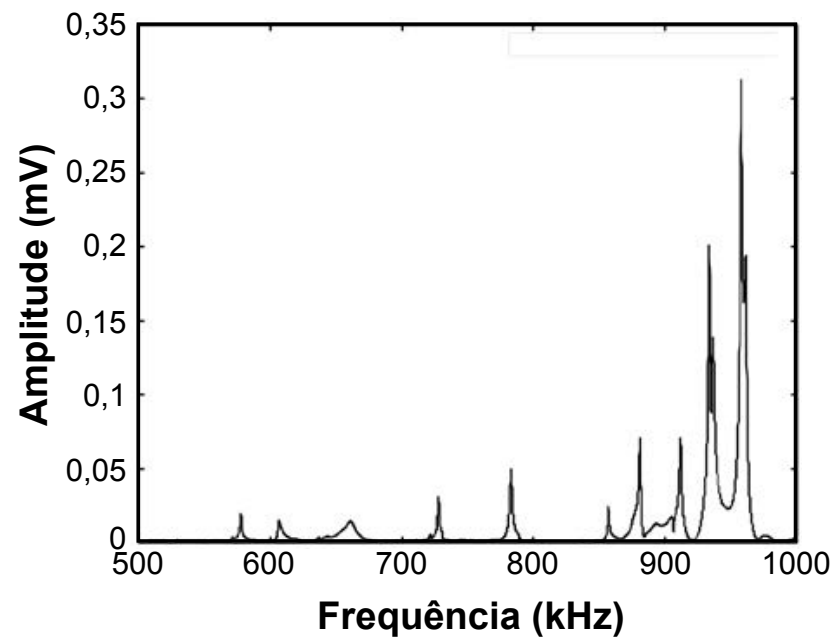

Figura 8: Espectro de ressonâncias para a amostra de níquel policristalino.

[Figure 8: Resonance spectrum for the sample of polycrystalline nickel.]

Os valores esperados das constantes elásticas $\mathrm{c}_{11}$ e $\mathrm{c}_{12}$ do níquel policristalino foram obtidos através da aproximação de Hashin e Strikman [3], partindo das constantes elásticas de um monocristal de níquel $\left(\mathrm{c}_{11}=261 \mathrm{GPa}, \mathrm{c}_{12}=151 \mathrm{GPa}\right.$, $\left.\mathrm{c}_{44}=132 \mathrm{GPa}\right)[20,21]$. Os resultados, resumidos na Tabela III, demonstram a excelente concordância entre os resultados experimentais e os valores esperados.

\section{CONCLUSÕES}

Foi descrita a montagem de um dispositivo para medidas de espectroscopia de ressonância de ultrassom. Testes realizados com amostras policristalinas de alumina e níquel apresentaram resultados em acordo com os valores da literatura. Apesar do procedimento relativamente complexo de cálculo das constantes elásticas a partir das freqüências de ressonância da amostra, a simplicidade do arranjo experimental, a pequena dimensão da amostra e a possibilidade da determinação simultânea de todo o tensor de elasticidade, tornam esta técnica extremamente interessante no estudo do comportamento linear elástico de materiais.

\section{AGRADECIMENTOS}

Os autores agradecem a Jon B. Betts (Los Alamos National Laboratory, USA) pelo programa LabView para controle e aquisição de dados do RUS e, também, o apoio financeiro da FAPERGS, CAPES e CNPq para a realização deste trabalho.

\section{REFERÊNCIAS}

[1] P. Ravindran, L. Fast, P. A. Korzhavyi, B. Johansson, J. Wills, O. Eriksson, J. Appl. Phys. 84, 9 (1998) 4891-4904.

[2] C. B. Carter, M. G. Norton, Ceramic Materials - Sci. Eng. Springer, NY (2007).

[3] D. J. Green, "An Introduction to the Mechanical
Properties of Ceramics", Cambridge University Press, Cambridge, UK (1998).

[4] J. B. Wachtman, W. R. Cannon, J. M. Matthewson, "Mechanical Properties of Ceramics", 2a Ed., John Wiley \& Sons, Hoboken, NJ (2009).

[5] M. Levy, H. E. Bass, R. Stern, "Modern Acoustical Techniques for the Measurement of Mechanical Properties", 39 Academic Press, USA, (2001).

[6] R. F. de L. Lorenzi, "Montagem de um dispositivo para espectroscopia de ressonância de ultrassom e aplicação na análise da relaxação estrutural da fase amorfa do tungstato de zircônio”, Diss. Mestrado, PGMAT-UCS (2012).

[7] J. D. Maynard, Phys. Today 49, 1 (1996) 26-31.

[8] A. Migliori, J. L. Sarrao, "Resonant Ultrasound Spectroscopy: Aplications to Physics, Materials Measurements and Nondestructive Evaluation", John Wiley \& Sons, USA (1997).

[9] M. Radovic, E. Lara-Curzio, L. Riester, Mater. Sci. Eng. A 368 (2004) 56-70.

[10] A. Migliori, J. L. Sarrao, W. M. Visscher, T. M. Bell, M. Lei, Z. Fisk, R. G. Leisure, Physica B 183 (1993) 1-24.

[11] R. B. Schwarz, J. F. Vuorinen, J. Alloys Comp. 310 (2000) 243-250.

[12] Disponível em http://www.thinksrs.com/downloads/ PDFs/Catalog/SR844c.pdf. Acesso em 17 de janeiro de 2012.

[13] Disponível em http://www.thinksrs.com/downloads/ PDFs/Catalog/DS345c.pdf. Acesso em 17 de janeiro de 2012.

[14] J. Catafesta, R. Andreola, C. A. Perottoni, J. E. Zorzi, Cerâmica 53, 325 (2007) 29-34.

[15] A. Migliori, J. D. Maynard, Rev. Sci. Instrum. 76 (2005) 121301.

[16] S. Desgreniers, K. Lagarec, J. Appl. Crystallogr. 27 (1994) 432-434.

[17] Disponível em http://www.magnet.fsu.edu/ inhouseresearch/rus/index.html. Acesso em 11 de novembro de 2013.

[18] Disponível em http://www.ceramics.nist.gov/srd/ summary/A12O3.htm. Acesso em 04 de novembro de 2011. [19] J. S. Reed, "Principles of Ceramics Processing", $2^{\text {nd }}$ Ed., John Wileyand Sons, Inc., NY (1995).

[20] G. Simons, H. Wang, "Single Crystal Elastic Constants and Calculated Aggregate Properties", MIT Press, Cambridge, MA (1977).

[21] J. R. Neighbours, F. W. Bratten, C. S. Smith, J. Appl. Phys. 23 (1952) 389-393.

(Rec. 17/12/2013, Rev. 20/01/2014, 19/02/2014, Ac. $31 / 03 / 2014)$

\section{APÊNDICE}

Exemplo de arquivo de entrada e de saída para o cálculo das constantes elásticas independentes de uma amostra policristalina de alumina. No arquivo de entrada, as primeiras quatro linhas informam a respeito da classe de simetria do material que constitui a amostra, além das 
dimensões físicas e a massa da amostra. As freqüencias de ressonância medidas experimentalmente são informadas a partir da quinta linha do arquivo. Uma descrição mais detalhada do arquivo de entrada para o cálculo das constantes elásticas a partir de resultados de espectroscopia de ressonância de ultrassom se encontra na Ref. [6].

\section{ARQUIVOS DE ENTRADA}
Alumina Isotropic
4.61 .6
$0.24560 .2412 \quad 0.2079$
0. 0.0 .
1.378640 .1 .00
1.514840 .1 .00
1.613110 .1 .00
0.1 .00
1.682060 .1 .00
1.736200 .1 .00
1.752160 .1 .00
1.756700 .1 .00

$\begin{array}{llll}2 & 0140 & 0.04809 & 1.001\end{array}$

\section{ARQUIVOS DE SAÍDA}

Alumina Isotropic

free moduli are $\mathrm{c} 11, \mathrm{c} 44$

using 14 order polynomials

mass $=0.0481 \mathrm{gm}$ rho $=3.905 \mathrm{gm} / \mathrm{cc}$

$\mathrm{n} \quad$ fexfr $\quad \%$ errwt $\mathrm{k}$ i $\mathrm{df} / \mathrm{d}($ moduli)

$\begin{array}{lllllllll}1 & 0.000000 & 1.119795 & 0.00 & 0.00 & 4 & 1 & 0.00 & 1.00\end{array}$

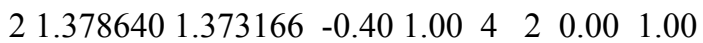

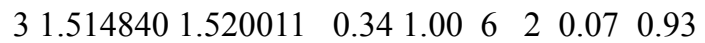

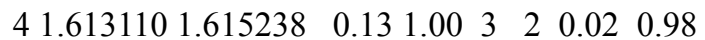

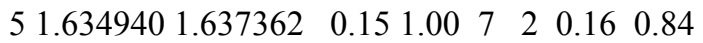

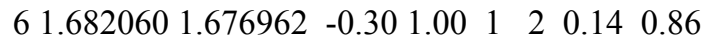

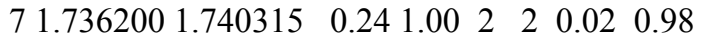

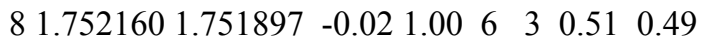

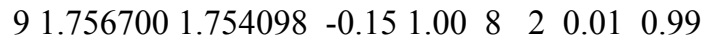

Bulk Modulus $=2.4463$

$\begin{array}{lllllllll}\text { c11 } & \text { c22 } & \text { c33 } & \text { c23 } & \text { c13 } & \text { c12 } & \text { c44 } & \text { c55 } & \text { c66 }\end{array}$ $\begin{array}{lllllll}4.52366 & 4.52366 & 4.52366 & 1.40758 & 1.40758 & 1.40758\end{array}$ $1.55804 \quad 1.558041 .55804$

d1 $\quad$ d $2 \quad$ d 3

$\begin{array}{lll}0.24560 & 0.24120 & 0.20790\end{array}$

loop\# $3 \mathrm{rms}$ error $=0.2459 \%$, changed by $-.0001729 \%$ 